\title{
Avant-propos
}

La mécanique quantique est une branche de la physique dont l'importance n'a cessé de s'accroître au cours des dernières décennies. Elle est bien sûr essentielle pour comprendre la structure et la dynamique des objets microscopiques comme les atomes, les molécules, ainsi que leurs interactions avec le rayonnement électromagnétique. Mais elle est aussi à la base du fonctionnement de nombreux systèmes nouveaux comme les sources laser (communications, médecine, usinage, etc.), les horloges atomiques (essentielles, en particulier, pour le GPS), les transistors (et donc les communications, l'informatique), l'imagerie par résonance magnétique, la production d'énergie (capteurs solaires, nucléaire), etc., donc des applications pratiques innombrables. Elle permet également d'expliquer des phénomènes surprenants comme la superfluidité ou la supraconductivité. Un grand intérêt est actuellement porté aux états quantiques intriqués, dont les propriétés de non-localité et non-séparabilité sont peu intuitives, et permettent d'envisager des applications remarquables dans le domaine de l'information quantique. Notre civilisation devient ainsi de plus en plus imprégnée par les applications technologiques qui découlent des concepts quantiques. Il est par suite clair qu'une attention particulière doit être portée à l'enseignement de la mécanique quantique. L'objet de ces trois tomes est de concourir à cet objectif.

Un premier contact avec la mécanique quantique peut cependant être très déroutant. Le présent ouvrage, issu de plusieurs enseignements auprès des étudiants, a été conçu dans le but de faciliter une approche initiale, et d'aider ensuite le lecteur à progresser continûment vers un niveau avancé de mécanique quantique. Les deux premiers tomes, publiés il y a plus de 40 ans, ont été utilisés dans le monde entier et traduits dans de multiples langues. Ils restaient toutefois à un niveau intermédiaire; l'ouvrage est maintenant complété par un troisième tome qui permet au lecteur d'aller plus loin. L'ensemble est systématiquement fondé sur une approche progressive des problèmes, où aucune difficulté n'est passée sous silence, et où chaque aspect des diverses questions est discuté en détail (en partant souvent d'un rappel classique).

Cette volonté d'aller au fond des choses « sans tricher ni prendre de raccourci» se concrétise dans la structure même de l'ouvrage, construite à l'aide de deux textes distincts mais imbriqués : les chapitres et les compléments. Les chapitres se suivent pour présenter les idées générales et les notions de base. Chaque chapitre est suivi de plusieurs compléments, en nombre variable, qui illustrent les méthodes et concepts qui viennent d'être introduits. Les compléments sont indépendants les uns des autres, et leur but est de proposer un large éventail d'applications diverses et prolongements intéressants. Pour faciliter l'orientation du lecteur et lui permettre d'organiser ses lectures successives, un guide de lecture comprenant une liste de commentaires des compléments un par un est proposée à la fin de chaque chapitre.

Le tome I présente une introduction générale du sujet, suivie d'un chapitre détaillé qui décrit les outils mathématiques de base de la mécanique quantique. Ce chapitre peut paraître un peu long et dense, mais l'expérience d'enseignement des auteurs a montré que cette présentation est à terme la plus efficace. Les postulats sont clairement énoncés à partir du troisième chapitre, avec de nombreuses illustrations en compléments. Ensuite sont décrites quelques grandes applications de la 
mécanique quantique, par exemple l'oscillateur harmonique, qui donne lieu à de très nombreuses applications (vibration des molécules, phonons, etc.), dont bon nombre font l'objet d'un complément spécifique.

Le tome II poursuit dans cette voie, en élargissant sa portée, et à un niveau un peu plus élevé. Il aborde la théorie des collisions, le spin, la composition des moments cinétiques et les calculs des perturbations indépendantes ou dépendantes du temps. Il fait une première incursion dans l'étude des particules identiques. Dans ce tome, comme dans le précédent, toute notion théorique est immédiatement illustrée par des applications diverses présentées dans des compléments. Comme le tome I, il a bénéficié de quelques corrections récentes, mais il a également été augmenté : le chapitre XIII comprend maintenant deux $\S \S \mathrm{D}$ et $\mathrm{E}$ qui traitent des perturbations aléatoires, et un complément entier sur la relaxation a été ajouté à ce chapitre.

Enfin le tome III vient maintenant compléter les deux premiers, en se situant à un niveau plus élaboré. Il se base sur l'usage du formalisme des opérateurs de création et d'annihilation (deuxième quantification), d'utilisation courante en théorie quantique des champs. Dans une première partie, on étudie les systèmes de particules identiques, fermions et bosons. Les propriétés des gaz parfaits en équilibre thermique sont exposées. Pour les fermions, la méthode de Hartree-Fock est présentée en détail ; elle est à la base d'un nombre considérable d'études en chimie, physique atomique ou du solide, etc. Pour les bosons, l'équation de Gross-Pitaevskii et la théorie de Bogolubov sont discutées. Une présentation originale qui regroupe les effets d'appariement dans les fermions et les bosons permet d'obtenir la théorie BCS (Bardeen-Cooper-Schrieffer) et de Bogolubov dans un cadre unifié. Une seconde partie du tome III est consacrée à l'électrodynamique quantique, son introduction générale, l'étude des interactions entre atomes et photons, et diverses applications (émission spontanée, transitions multi-photoniques, pompage optique, etc.). La méthode de l'atome habillé est présentée et illustrée dans des cas concrets. Un dernier chapitre discute la notion d'intrication quantique et certains aspects fondamentaux de la mécanique quantique, en particulier les inégalités de Bell et leur violation.

Mentionnons enfin que nous n'avons abordé, ni la discussion des implications philosophiques de la mécanique quantique, ni celle des diverses interprétations de cette théorie, malgré le très grand intérêt qui s'attache à ces sujets. Nous nous sommes en fait limités à présenter que l'on appelle souvent "le point de vue orthodoxe", et seul le Chapitre XXI s'approche un peu de certaines questions touchant les fondements de la mécanique quantique (sa non-localité, etc.). Nous avons fait ce choix car il nous semble que l'on peut s'intésser aux questions relatives aux fondements de façon plus efficace une fois que l'on a acquis au préalable une bonne aisance dans le maniement pratique de la mécanique quantique, ainsi que de ses applications si nombreuses. Ces sujets sont abordés dans l'ouvrage Comprenons-nous vraiment la Mécanique Quantique? (F. Laloë, EDP Sciences, 2017) ; voir également la section 5 de la bibliographie des Tomes I et II. 


\section{Remerciements :}

Tomes I et II : l'enseignement qui est à l'origine de cet ouvrage résulte d'un travail d'équipe qui s'est poursuivi pendant plusieurs années.Nous tenons à remercier ici tous les membres des diverses équipes dont nous avons fait partie, et tout particulièrement Jacques Dupont-Roc et Serge Haroche, pour leur collaboration amicale, les discussions fructueuses que nous avons eues ensemble lors de nos réunions hebdomadaires, les idées de problèmes et d'exercices qu'ils nous ont suggérées. Sans leur enthousiasme et leur aide précieuse, nous n'aurions jamais pu entreprendre et mener à bien la rédaction de cet ouvrage. Nous ne saurions également oublier tout ce que nous devons aux physiciens qui nous ont initiés à la recherche, Alfred Kastler et Jean Brossel pour deux d'entre nous, Maurice Lévy pour le troisième. C'est dans l'ambiance de leurs laboratoires que nous avons découvert la beauté et la puissance de la mécanique quantique. Nous n'oublions pas non plus l'importance qu'a eue pour nous l'enseignement de la physique moderne dispensé au C.E.A. par Albert Messiah, Claude Bloch et Anatole Abragam, à une époque où le troisième cycle n'avait pas encore fait son apparition dans l'enseignement supérieur.

Tome III : Nicole et Dan Ostrowsky ont, à l'occasion de leur traduction du texte en anglais, proposé de nombreuses améliorations ou clarifications, et nous leur en sommes très reconnaissants. Nombreux sont en fait les collègues et amis qui ont grandement contribué à la mise au point de cet ouvrage. Cela nous a d'autant plus aidés que chacun, dans son style propre, nous a apporté des remarques et suggestions complémentaires, et toujours utiles. Tous nos remerciements vont donc en particulier à :

Pierre-François Cohadon

Jean Dalibard

Sébastien Gleyzes

Markus Holzmann

Thibaut Jacqmin

Philippe Jacquier

Amaury Mouchet

Jean-Michel Raimond

Félix Werner

De plus, Marco Picco et Pierre Cladé nous ont grandement aidés à maîtriser certains aspects délicats de la typographie Latex, et à vectoriser des figures. Roger Balian, Edouard Brézin et William Mullin nous ont fait bénéficier d'utiles conseils et suggestions. Enfin, pour un certain nombre de figures, nous remercions vivement pour leur aide Geneviève Tastevin, Pierre-François Cohadon et Samuel Deléglise. 